\title{
Damage due to rolling in total knee replacement-The influence of tractive force
}

\author{
Markus A. WIMMER ${ }^{1, *}$, Lars BIRKEN ${ }^{2}$, Kay SELLENSCHLOH ${ }^{2}$, Erich SCHNEIDER ${ }^{2}$ \\ ${ }^{1}$ Section of Tribology, Rush University Medical Center, Chicago, IL 60612, USA \\ ${ }^{2}$ Section of Biomechanics, Hamburg University of Technology, Hamburg, 21071, Germany \\ Received: 05 March 2013 / Revised: 30 April 2013 / Accepted: 20 May 2013 \\ C The author(s) 2013. This article is published with open access at Springerlink.com
}

\begin{abstract}
The femoral condyles of a knee prosthesis articulate with a combination of rolling and sliding on the tibial polyethylene plateau. Little is known about potential polyethylene damage due to rolling motion. Since rolling does not exclude the presence of tangential surface loads, this study sought to investigate the influence of tractive rolling on the wear of polyethylene. A "wheel-on-flat" apparatus, consisting of a metal wheel and a polyethylene flat, mimicked contact conditions present in total knee replacement. An increasingly tractive force under conditions of pure rolling was applied. It was found that under rolling kinematics a tangential surface load of up to $17 \%$ of the normal load could be transferred through the contact. Surface damage was dependent on the amount of tractive force and appeared more severe with higher forces. In the region of highest tractive force, wear features were identified that resembled perpendicular ridges on surfaces of retrieved tibial polyethylene devices. This suggests that tractive rolling may be a relevant wear mode in total knee replacement.
\end{abstract}

Keywords: polyethylene; wear; knee prosthesis; tractive rolling

\section{Introduction}

The knee is the largest and one of the most mechanically complex joints in the human body. The medial and lateral condyles of the distal part of the femur articulate against the medial and lateral compartments of the tibial plateau, similarly to cylindrical wheels on a flat surface. The primary motion of the knee joint is flexion-extension, i.e., the rotation around the "epicondylar axis" of the femoral condyles, but secondary motions, as for example translations on the tibial plateau, occur as well. Hence, the kinematic interaction of the surfaces is best described as a rollingsliding action. Modern, successful designs in total knee replacement mimic the anatomy of the natural joint and allow the same degrees of freedom. Typically, the surfaces of the femoral condyles are replaced with metal that articulate against polyethylene anchored

\footnotetext{
* Corresponding author: Markus A. WIMMER.

E-mail: Markus_A_Wimmer@rush.edu
}

on the tibial plateau. This polyethylene component is prone to wear and has been subject of many investigations since wear jeopardizes the longevity of the implant.

In many studies, cyclic sliding has been assumed to be the most relevant kinematic action in the generation of polyethylene wear debris and was investigated using a variety of test set-ups [1-3]. Blunn et al. [4] were the first to investigate the influence of rolling versus sliding. For this purpose, they modified a reciprocating pin-on flat device to provide rolling and sliding under cyclic load. The pin was replaced with a polished sphere that rolled or slid over the flat UHMWPE disc. To produce rolling, the "tibial" disc was reciprocated and the "femoral" sphere was rotated in synchrony. To achieve sliding, the sphere was blocked. Rolling resulted in the generation of shallow wear tracks without major damage, while sliding produced deeper impressions with evidence of subsurface cracking. The reduced amount of wear during rolling was attributed to the lack of frictional shear forces across the surface. 
Using the terminology introduced by Johnson [5], "free rolling" had been applied in the experiment mentioned above. Under conditions of free rolling, tangential surface loads are zero. "Tractive rolling", in contrast, describes dynamic conditions where tangential surface loads are present under pure rolling conditions. In total knee replacements, tractive forces can be substantial and it has been estimated that they can reach up to $20 \%$ of the contact load during walking activity [6]. The purpose of this study was to experimentally study the traction coefficient, i.e., the ratio of tractive to normal force during rolling motion, and to investigate the influence of increasing tractive force on surface wear.

\section{Materials \& methods}

A special testing apparatus was designed to simulate increasing tractive forces under conditions of pure rolling. The oscillating "wheel-on-flat" configuration consists of a wheel that drives a polyethylene (PE) flat with increasing tractive force against a pneumatic cylinder (Figs. 1(a) and 1(b)).

The following describes one full operating cycle: After wheel and PE flat are in starting position and the desired constant normal load is reached, the wheel starts turning at constant angular velocity. It drives the PE flat, which is mounted on a sledge supported by roller bearings, against a tangential cylinder. The force in the cylinder is regulated via closed loop control and increases linearly with sledge displacement.

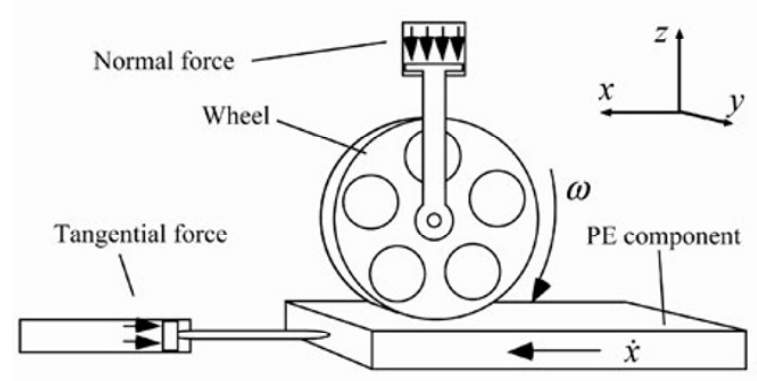

(a)
Thus, an increasing tractive force at the rolling contact between wheel and flat is generated. Tournament of the wheel and cylinder force are stopped immediately when sliding is detected. Wheel and PE flat are then separated from each other and set back to the starting positions to begin a new cycle. This was done in an attempt to avoid the superposition of surface features, which would have been generated during the return stroke of wheel and PE flat.

The testing apparatus was designed to provide easy access to the articulating components, the wheel and the PE flat (Fig. 1(b)). The sledge is supported by high precision bearings underneath, which account for negligible friction forces (3-5 N under $1000 \mathrm{~N}$ of normal load). A pneumatic short-stroke cylinder, driven by a $3 / 2$ electric directional control valve and a pressure regulator, supplies the normal load in order to bring wheel and flat into contact. The wheel itself is driven by a pneumatic rotary actuator. Both, wheel and actuator are mounted onto a frame being constrained to $z$-motion and assuring that the rotational axis of the wheel runs parallel to the $y$-axis of the sledge (Fig. 1(a)). This arrangement ensures correct alignment of the cylindrical wheel on the polyethylene surface and compensates irregularities within the articulation. A tensile spring supports the weight of the free-hanging wheel and actuator unit. A high precision valve, designed for closed loop control, adjusts the air pressure in the rotary cylinder. Two additional $3 / 2$ directional control valves on each side of the doubleacting cylinder are used for quick air release. This

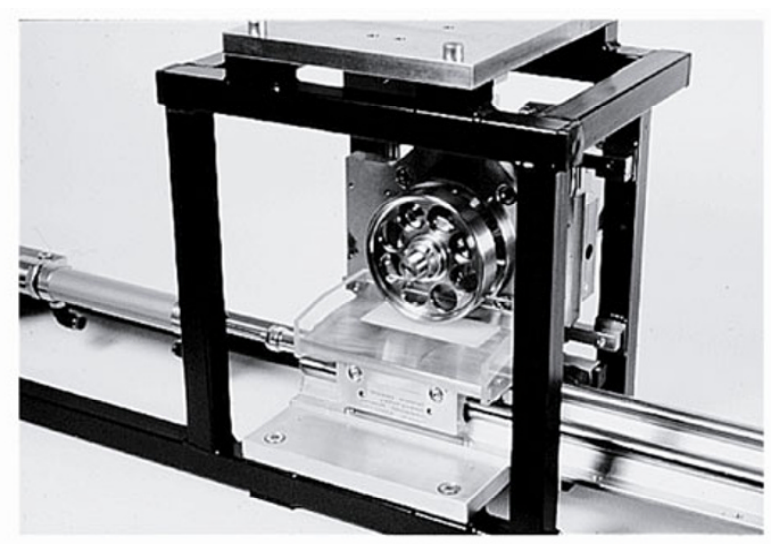

(b)

Fig. 1 (a) Mechanical concept of the "wheel-on-flat" apparatus: the sledge is driven by the wheel against a tangential force, actively produced by a pneumatic cylinder. (b) Photograph of the wear testing device. 
allows for a quick stop of the system once sliding is detected. An identical arrangement of valves was applied to the linear cylinder which provides the tangential force to the sledge.

Ultra-high molecular weight polyethylene (UHMWPE) flats in dimensions of $100 \mathrm{~mm} \times 40 \mathrm{~mm} \times 8 \mathrm{~mm}$ were compression molded using Himont 1900 powder by Zimmer Inc. (Warsaw, IN, USA). UHMWPE powder, pressure, and temperature protocols were similar to those used for Miller-Galante knee inserts and, thus, facilitated the comparison to retrieved components of such design. Cylindrical wheels of $100 \mathrm{~mm}$ in diameter and $20 \mathrm{~mm}$ in width were manufactured from cast cobalt-chromium-molybdenum alloy (CoCrMo) according to ASTM F75 by Implantcast $\mathrm{GmbH}$ (Buxtehude, Germany). A polishing surface finish similar to commercially available prostheses was applied $\left(R_{\mathrm{a}}=0.04 \mu \mathrm{m}\right)$.

Three experiments were executed at room temperature using the compressive force mode of the tangential cylinder without lubricant. This was done in an attempt to achieve worst case conditions and the highest tractive forces possible. A constant normal force of $900 \mathrm{~N}$ was applied to the wheel. This reflects about $50 \%$ of the average normal load during stance phase of gait of a $60-75 \mathrm{~kg}$ person (half of the load is applied because only one of the two femoral condyles is modeled in this wear test). The load characteristics of the tangential cylinder were adjusted so the load increased linearly with $2.3 \mathrm{~N} / \mathrm{mm}$ starting from zero. Thus, the length of the wear track covered at least $70 \mathrm{~mm}$ before the force got too high for pure rolling. This approach made it possible to relate specific locations on the polyethylene flat to precise loading characteristics. 0.5 million cycles were performed. Each pass over the PE flat lasted approximately $1 \mathrm{~s}$. All tests were run under a positive pressure hood to keep contamination to a minimum. Throughout the experiment, tractive force $F_{\mathrm{t}}$ (sensor range $500 \mathrm{~N}$, error $< \pm 0.1 \%)$, normal force $F_{\mathrm{n}}(2 \mathrm{kN}, \pm 0.5 \%)$, PE flat displacement $(100 \mathrm{~mm}, \pm 0.1 \%)$, and wheel motion $\left(120^{\circ}, \pm 0.05 \%\right)$ were measured. The data acquisition was set to $150 \mathrm{~Hz}$ per channel and measurements were taken every 5000 cycles for an interval of 5 cycles. Thereby, the digital resolution was set to approx. $1 \%$ of the measured values. The traction coefficient $\mu_{\mathrm{t}}=F_{\mathrm{t}} / F_{\mathrm{n}}$ was calculated from the determined maximum tractive force $F_{\mathrm{t}}$ during rolling movement.

At the end of each wear test, CoCrMo wheel and PE flat were rinsed with distilled water to remove any loose wear particles from the surfaces. Then, the specimens were dried for $24 \mathrm{~h}$. Initially, wear characteristics of wheel and PE flat were analyzed and mapped under polarized contrast using a light microscope (Orthoplan, Leitz, Germany). Afterwards, to obtain more detail of characteristic sections, a low voltage scanning electron microscope (LVSEM: S-4500, Hitachi, Japan) was used. No conductive coating was applied during this initial stage of the investigation. Later, samples were carbon sputtered to allow energy dispersive spectroscopy at $15 \mathrm{kV}$ (Link ISIS, Oxford Instruments, England) and to identify any system inherent or extrinsic contaminants which could have influenced the wear process.

\section{Results}

As intended, rolling motion of the wheel was initiated without major tractive force on the polyethylene flat, but plastic deformation at the resting position created constrained forces. This raised the initial force reading to approximately 25-50 N. Once this dip was cleared, the tractive force increased as a linear function of sledge displacement, however, plotted as a time function, the behavior was non-linear (Fig. 2).

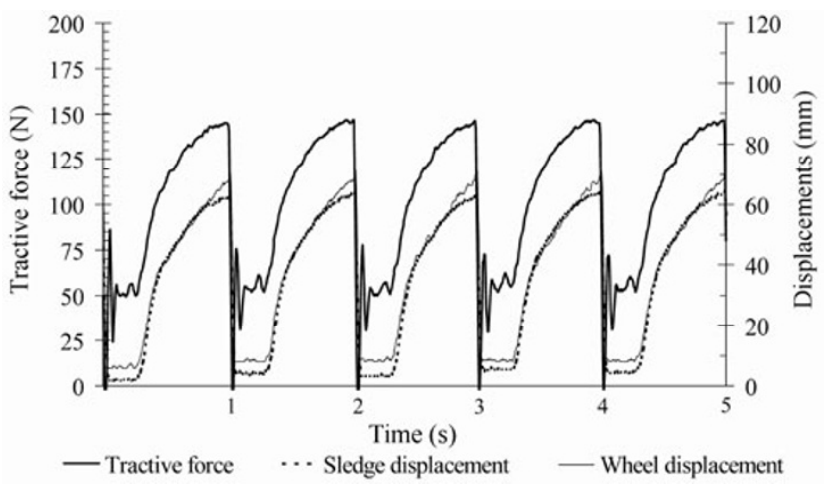

Fig. 2 Five consecutive cycles showing the tractive force, sledge displacement and wheel displacement. Note that the velocity of the driving wheel was constant during the first $35 \mathrm{~mm}$ of displacement and then diminished. A time relay, effective at cycle start, delayed motion initiation until vibrations due to air filling of cylinders faded. In this example, the surface deformation at start was large enough to create an offset in force reading (see text). 
This non-linearity was caused by deceleration of the wheel due to an increasing traction moment with sledge displacement. Maximum traction coefficients yielded 0.13 to 0.17 and were maintained throughout testing. The transition from rolling to sliding was abrupt, but did not cause any instability in the control algorithm.

The CoCrMo wheel showed only minor surface damage to the unaided eye after testing. Under the microscope, uni-directional, mild scratching was observed (Fig. 3). For all tested wheels, scratches looked similarly throughout the wear track on the metal surfaces, i.e., an influence of tractive force was not identified. Using LVSEM, it was found that the scratches were up to $0.6 \mu \mathrm{m}$ in width. The smooth appearance suggested a micro-plowing rather than a micro-cutting mechanism. No foreign material could be identified inside the scratches.

Repeated rolling of the wheel across the PE flat generated a macroscopically visible deformed path, mostly from plastic deformation of the polyethylene material. The severity of damage on the polyethylene surface increased with increasing tractive force (Fig. 4).

The whole wear track of the polyethylene plateau was covered with fine, longitudinal scratches following the direction of motion. Using microscopy, no differences in morphological appearance could be found along the course. Also, pitting and transferred polyethylene particles were observed over the whole

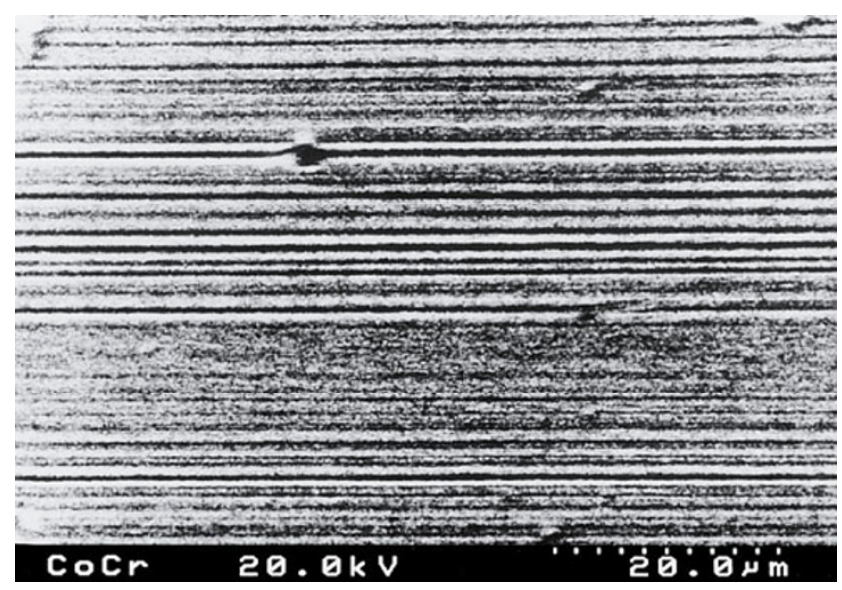

Fig. 3 Fine scratches on the CoCrMo wheel that were aligned in direction of motion.
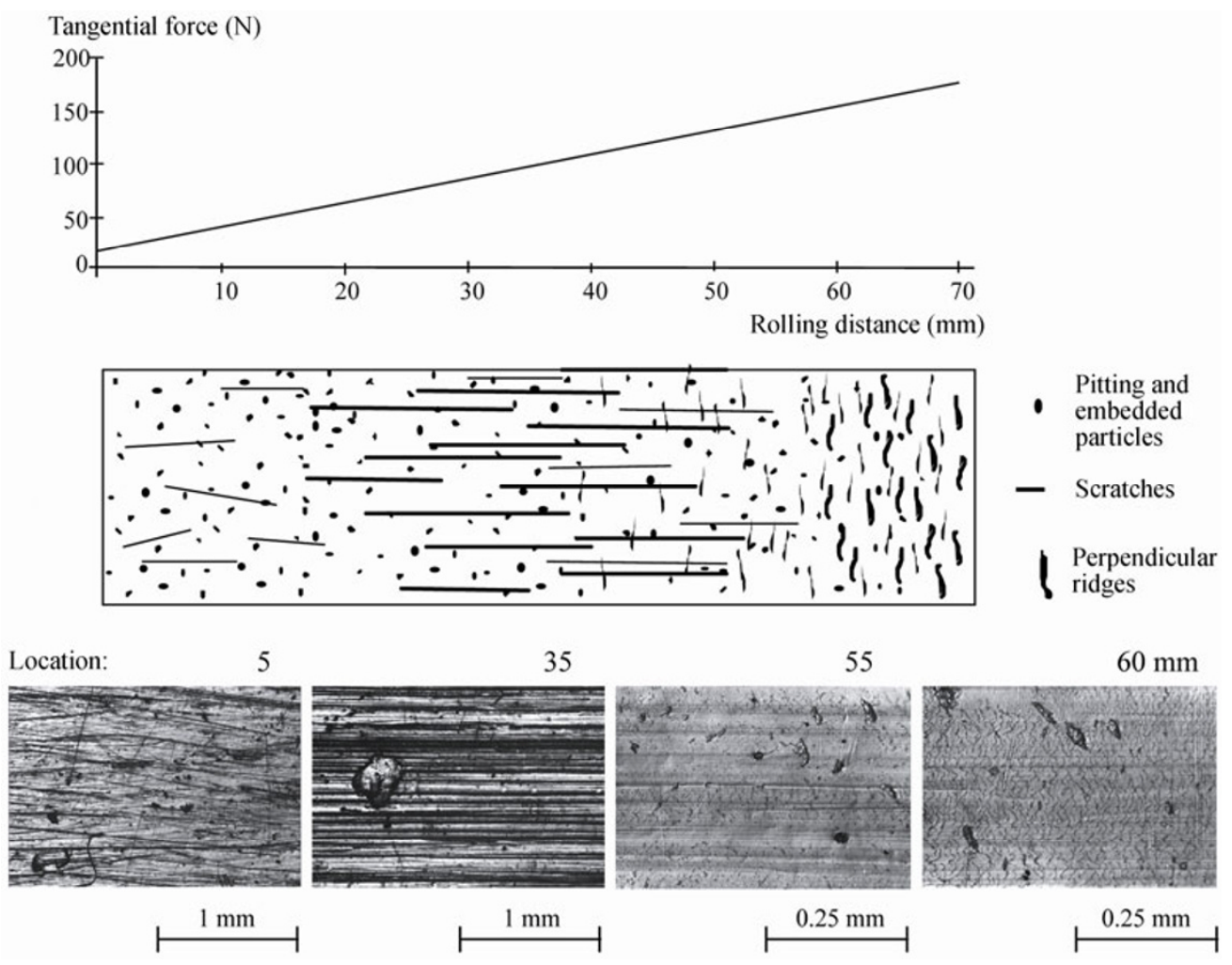

Fig. 4 The appearance of wear on the tibial plateau changes with applied tractive force. The wheel moved from left to right over the PE flat. 
wear track. Pit sizes were usually confined to a diameter of 10 to $20 \mu \mathrm{m}$, while re-embedded particles could gain several hundred microns in width. Longitudinal scratching also occurred, however, its appearance varied with location on the wear track. Within the first $30 \mathrm{~mm}$ of the wear track, random oriented scratches deviating $0^{\circ}$ to $20^{\circ}$ from the principal direction of motion were found. From 30 to $50 \mathrm{~mm}$, these scratches became more indented and more oriented (in the direction of movement). Within the last $20 \mathrm{~mm}$ of the wear track, scratching as a damage feature disappeared and another wear feature occurred: ridges perpendicular to the direction of motion came into view, pronounced in height and frequency within the last few millimeters of the wear track. In that area the ridges reached about 5 to $10 \mu \mathrm{m}$ in length and seemed to be built up rather than separated from the surface (Fig. 5). Loose particles with varying sizes, rarely exceeding $1 \mu \mathrm{m}$, were found. After carbon coating, the particles were analyzed. Their elemental composition was determined to sodium, potassium and chlorine. In some cases, calcium and phosphor were identified, too.

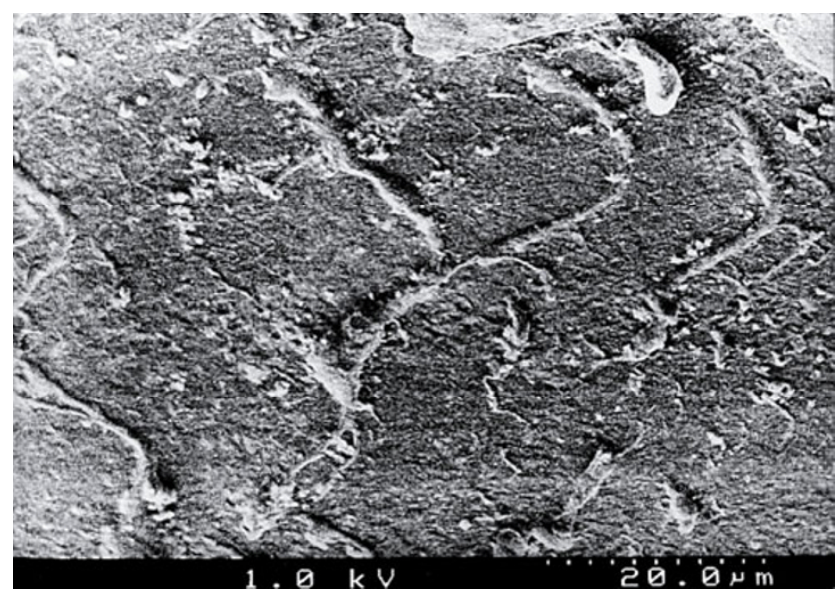

Fig. 5 A close-up of the perpendicular ridges occurring at locations of highest tractive force at the tibial plateau.

\section{Discussion}

This study attempted to relate tractive rolling contact conditions of the knee joint to the appearance of surface wear of the contacting bodies. The specific approach, namely to control the tractive force at the interface by contact displacement (rather than time), allowed to classify the observed wear features relative to the tractive force magnitude. In addition, the traction coefficient during rolling motion of CoCrMo alloy on UHMWPE was studied.

The coefficient of traction is an important factor influencing the kinematics of the tibio-femoral articulation [6]. Once the maximum traction coefficient is reached, rolling stops and gross sliding takes place. In this study, maximal traction coefficients of 0.17 occurred. Since no lubricant was used in this study, the value can be considered as the upper limit in total knee replacement. Under lubricated conditions the coefficient should be lower; however, as will be shown below, damage features that occurred under high tractive force in this study were also present on retrieved tibial polyethylene components. This is an indication that tractive rolling is occurring in artificial knee joints. These kinematic characteristics are in contrast to the natural knee where friction is 10 to 100-fold lower [7] and the tractive force negligible.

Tractive rolling facilitates antero-posterior translation of the femoral condyles on the tibial polyethylene plateau in the absence of cruciate ligaments ${ }^{1}$; thus, contributing to femoral rollback of the artificial joint. Femoral rollback is an important mechanism to enable proper knee mechanics. It increases the lever arm between contact point (i.e., the instantaneous center of knee rotation) and extensor muscles during flexion maneuvers as for example during seating onto and rising from a chair. This minimizes the necessary muscle force to flex and extend the knee. Hence, femoral rollback comprises an important functional feature necessary for easy execution of daily activities. In the light of this discussion, a recent manuscript about a new type of a rolling artificial knee joint that has been based on the mechanics of rolling friction is noteworthy [9].

Figure 4 shows the wear appearances of the whole track generated due to tractive rolling. Note that the different damage features are concentrated in certain areas of the PE flat and, thus, are related to tractive

\footnotetext{
${ }^{1}$ In the natural knee joint, the cruciate ligaments form a crossed four-bar linkage facilitating femoral rollback [8]. One or two ligaments are surgically removed during total knee arthroplasty destroying the linkage.
} 
force. Scratching is present along most of the wear track. Shallow, non-oriented scratches are found within the first $20 \mathrm{~mm}$ of the scar, where the tractive forces were low. Longitudinal scratches along the principal direction of motion are found thereafter and are related to tractive forces between 50 and $110 \mathrm{~N}$ (or traction coefficients from 0.06 to 0.12 ). These scratches are more pronounced and deeper than those at the beginning of the wear track. They seem to correspond in appearance and dimension to scratches found on the wheel suggesting third-body wear. Indeed, particles in a size were found matching the dimensions of the scratches. Their elemental composition points towards intrinsic salt contaminants occurring in UHMWPE [10].

Perpendicular ridges are present at the end of the wear track, where the tractive forces were highest. At least $100 \mathrm{~N}$ of tractive force (or a traction coefficient of 0.9 ) were necessary to produce ridges. However, their frequency increases and their appearance becomes more pronounced when the traction coefficient reached values beyond 0.12 . Due to the specific (moving) stress field, i.e., compressive stress in front and tensile stress behind the rolling wheel, high cyclic compressivetensile tangential stresses were induced to the polyethylene in that region of the wear scar [11]. Maximum shear stresses moved closer to the surface in that area, too. The applied stress field may have caused plastic deformation of near-surface polyethylene layers, thus leading to the formation of ridges perpendicular to the direction of tractive force. Plumlee and Schwartz [12], using finite element models in a recent study, found areas of plastic deformation within the metal-UHMWPE contact area that were of the same size scale as the ridges observed herein. It should be noted that partially released surface layers and perpendicular ridges are capable of forming particulate debris and/ or initiating progressive delamination if the material embrittles due to oxidation.

Ridges similar to those observed in this study were previously found on retrieved UHMWPE tibial plateaus using white light interferometry [13]. Interestingly, they are present on top of antero-posterior oriented "striations", which protrude from the polyethylene surface and form troughs in between (Fig. 6). On top of the striated hills, the ridges run perpendicular to

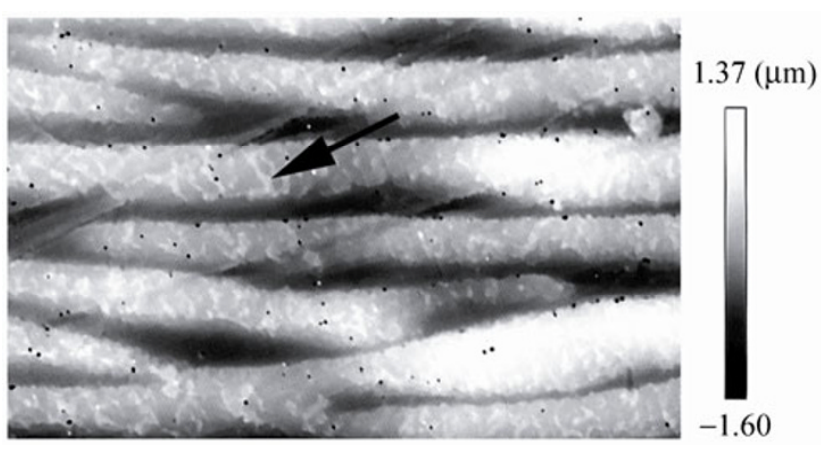

Fig. 6 Height image taken by white light interferometry. Note the perpendicular ridges (arrow) on the summits of the striated pattern.

the main direction of rolling (anerior-posterior). The striated pattern represents a typical wear pattern in total knee replacement and has been reported by several investigators [14-16].

Their specific feature is a topographical profile that has similarities to the threads of a vehicle rubber tire. It is therefore hypothesized that the striated pattern enables the drainage of lubricant through its troughs during rolling contact and thus facilitates high traction coefficients. The occurrence of the perpendicular ridges on top of this surface feature seems to provide indirect proof of this concept.

The study has several limitations which shall be briefly highlighted. The "wheel-on-flat" design is a very crude approximation of total knee prosthesis, which is much more complex in shape than a single radius contacting a flat surface. The studied motions are therefore not directly translatable to the interface of an artificial joint. The experiment was conducted under dry conditions at room temperature. This may be looked at as a worst case scenario, but under all likelihood the in vivo contact is wet, thus lowering the coefficient of friction and therefore the tractive force. Despite that, similar surface features produced in this study have been observed in vivo.

It should be mentioned that this is an early study which triggered follow-up studies from the same author group [17-19]. None of these studies, however, focuses on surface features as related to tractive force. In general, it is surprising how little scientific effort has been spent to disentangle the mechanistic interactions of sliding and rolling at the knee joint since 
the landmark study of Blunn et al. [4]. Two studies which further contributed to the field are from Cornwall et al. [20], who studied the three basic kinematic contact conditions (sliding, gliding, rolling) in a reciprocating wear tester, and from Keller et al. [21], who studied the microstructural re-organization of polyethylene after sliding-rolling contact.

\section{Conclusions}

Tractive rolling is a likely kinematic condition in total knee replacement. Tractive coefficients up to 0.17 may occur, stressing the polyethylene surface. This study demonstrated that the damage features due to rolling are dependent on tractive force and culminate in perpendicular ridges, most likely generated due to flow of near-surface polyethylene layers.

\section{Acknowledgements}

This work was conducted at the TUHH Hamburg University of Technology, Germany within the framework of a doctoral thesis [13].

Open Access: This article is distributed under the terms of the Creative Commons Attribution Noncommercial License which permits any noncommercial use, distribution, and reproduction in any medium, provided the original author(s) and source are credited.

\section{References}

[1] White S E, Whiteside L A, McCarthy D S, Anthony M, Poggie R A. Simulated knee wear with cobalt chromium and oxidized zirconium knee femoral components. Clin Orthop 309: 176-184 (1994)

[2] Blunn G W, Lilley A P, Walker P S. Variability of the wear of ultra high molecular weight polyethylene in simulated TKR. Trans Orthop Res Soc 19: 177 (1994)

[3] Walker P S, Ben-Dov M, Askew M J, Pugh J. The deformation and wear of plastic components in artificial knee joints-An experimental study. Eng Med 10: 33-38 (1981)

[4] Blunn G W, Walker P S, Joshi A, Hardinge K. The dominance of cyclic sliding in producing wear in total knee replacements. Clin Orthop 273: 253-260 (1991)

[5] Johnsen K L. Contact Mechanics, 2nd Editon. Cambridge (UK): Cambridge University Press, 1985.
[6] Wimmer M A, Andriacchi T P. Tractive forces during rolling motion of the knee: implications for wear in total knee replacement. J of Biomechanics 30: 131-137 (1997)

[7] Unsworth A. Lubrication of human joints. In Mechanics of Human Joints: Physiology, Pathophysiology, and Treatment. Wright V, Radin E L, Eds. New York: Marcel Dekker Inc., 1993: 137-162.

[8] O'Connor J J, Zavatsky A. Kinematics and mechanics of the cruciate ligaments of the knee. In Biomechanics of Diathrodial Joints, Vol II. Mow V C, Ratelitte A, Woo S L-Y, Eds. New York: Springer, 1990: 197-241.

[9] $\mathrm{He} \mathrm{Y,} \mathrm{Yu} \mathrm{Z,} \mathrm{Chen} \mathrm{M,} \mathrm{Wang} \mathrm{C.} \mathrm{Rolling} \mathrm{friction:} \mathrm{A} \mathrm{design}$ of artificial knee joint (in Chinese). Journal of Biomedical Engineering 22(4): 840-843 (2005)

[10] Loos J, Wimmer M A. Observation of salt impurities in ultrahigh-molecular-weight polyethylene (UHMWPE). Journal of Materials Science 34: 3327-3333 (1999)

[11] Natarajan R N, Hussain M, Wimmer M A, Rosenberg A G, Jacobs J J. Wear of UHMWPE total knee components of total knee replacement depend upon walking pattern. Trans Orthop Res Soc 29: 1035 (2004)

[12] Plumlee K G, Schwartz C J. Surface layer plastic deformation as a mechanism for UHMWPE wear, and its role in debris size. Wear, in press, http://dx.doi.org/10.1016/j.wear.2012.11.081.

[13] Wimmer M A. Wear of the polyethylene component created by rolling motion of the artificial knee joint. Ph.D. Thesis. Aachen (Germany): Technische Universitaet HamburgHarburg, 1999: 81-117.

[14] Wimmer M A, Andriacchi T P, Natarajan R N, Loos J, Karlhuber M, Petermann J, Schneider E, Rosenberg A G. A striated pattern of wear in ultrahigh-molecular-weight polyethylene components of Miller-Galante total knee arthroplasty. J Arthroplasty 13: 8-16 (1998)

[15] Harman M K, DesJardins J, Benson L, Banks S A, LaBerge M, Hodge W A. Comparison of polyethylene tibial insert damage from in vivo function and in vitro wear simulation. J Orthop Res 27(4): 540-548 (2009)

[16] Heyse T J, Davis J, Haas S B, Chen D X, Wright T M, Laskin R S. Retrieval analysis of femoral zirconium components in total knee arthroplasty: Preliminary results. $J$ Arthroplasty 26(3): 445-450 (2011)

[17] Schwenke T, Borgstede L L, Schneider E, Andriacchi T P, Wimmer M A. The influence of slip velocity on wear of total knee arthroplasty. Wear 259(7-12): 926-932 (2005)

[18] Galetz M C, Uth T, Wimmer M A, Adam P, Glatzel U. Determination of the temperature rise within UHMWPE tibial components during tribological loading. Acta Biomaterialia 6(2): 552-562 (2010) 
[19] Schwenke T, Wimmer M A. Cross-shear in metal-onpolyethylene articulation of orthopaedic implants and its relationship to wear. Wear, in press, http://dx.doi.org/10.1016/ j.wear.2013.01.069.

[20] Cornwall G B, Bryant J T, Hansson C M. The effect of kinematic conditions on the wear of ultra-high molecular

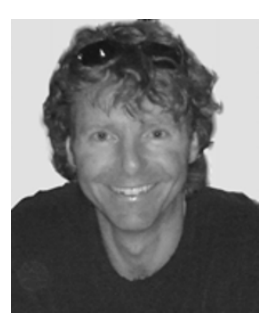

Markus A. WIMMER is an Associate Professor of orthopedics and directs the Motion Analysis and Tribology Laboratories at Rush University Medical Center. He is also an Adjunct Professor of bioengineering at the University of Illinois at Chicago. He studied mechanical engineering at the Technical University of Munich, Germany and received his diploma in 1992. After a post-graduate year in the laboratory of Dr. Thomas Andriacchi at Rush University weight polyethylene (UHMWPE) in orthopaedic bearing applications. Proc Inst Mech Eng H 215(1): 95-106 (2001)

[21] Keller T F, Engelhardt H, Adam P, Galetz M C, Glatzel U, Jandt K D. Near-surface microstructural reorganization of UHMWPE under cyclic load-A pilot study. Advance Engineering Materials 13(12): 476-482 (2011)

(Chicago, USA), he started as a Research Associate at the Hamburg University of Technology and worked towards a doctoral degree in biomechanics under the supervision of Dr. Erich Schneider. In 1997, Markus Wimmer moved from Germany to Switzerland and started to work at the AO Research Institute in Davos. He returned to Rush University in 2001 to join the faculty of the Medical College. His current research focuses on wear of natural and artificial joints and includes, among other topics, polyethylene damage in knee prostheses. 